\title{
Epigenetic Regulation of Gut Microbial Dysbiosis
}

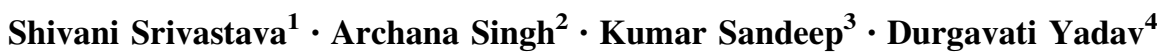

Received: 29 December 2020/ Accepted: 14 January 2021/Published online: 11 February 2021

(C) Association of Microbiologists of India 2021

\begin{abstract}
Microbiota inside the gut plays a vital role in maintaining human health. Microbial dysbiosis is associated with various complications leading to a range of diseases. Epigenetic changes enforced by various environmental and lifestyle factors lead to heritable modifications. These epigenetic modifications include DNA methylation, histone modifications, chromatin remodelling, and ribonucleic acid-based mechanisms. This review summarizes the impacts of environmental factors on the gut microbiome, epigenetic modifications, and their role in cardiovascular diseases.
\end{abstract}

Keywords Epigenetics - Dysbiosis · Human - Health · Disease $\cdot$ Microbiome $\cdot$ Microorganism

Kumar Sandeep

ksandeep83@gmail.com

$\triangle$ Durgavati Yadav

durgavati45yadav@gmail.com

1 Department of Physiology and Cell Biology, Wexner Medical Center, Davis Heart and Lung Research Institute, Ohio State University, Columbus, OH 43201, USA

2 Department of Bioinformatics, Banaras Hindu University, Mahila Mahavidyalaya, Varanasi 221005, India

3 Dr. B. R. Ambedkar Institute Rotary Cancer Hospital, All India Institute of Medical Sciences, New Delhi 110029, India

4 Department of Urology, All India Institute of Medical Sciences, New Delhi 110029, India

\section{Introduction}

Microbes including viruses, archaea, and bacteria live symbiotically on mucosal surfaces, mouth, gut, and skin of human beings. Around 1,000 unique species of microbes have been identified to survive in the human gut. The microbial colonization initiates from birth and maintains throughout the life span [1, 2]. Various studies have demonstrated important roles of microorganisms in diverse fields, such as fermentation [3, 4], biomolecules [5, 6], bioremediation [7-10], anti-toxicity [11, 12], and diseases and health [13-18]. Pathogenesis, including the recent outbreak of coronavirus, can be effectively treated through the application of these bioactive molecules, vaccines, and nanomaterials [11, 14, 19-24]. Microbiota coevolution with mammalian host results in the plethora of vital functions, such as metabolic signaling, energy metabolism, regulation of integrity, mobility of the gut barrier, and formation of the immune system. Gut microbiota plays a significant role in the manifestation of metabolic disorders and infections [1, 12, 25, 26].

International Human Epigenome Consortium and the Human Epigenome Projects have been initiated to understand the overall epigenetic mechanisms involved in human diseases and health. The changes in non-coding RNA were believed to cause obesity, diabetes, and neurodegenerative diseases and those affecting lung and liver $[27,28]$ (Fig. 1). The epigenetic DNA imprinting is usually the most active during the early 1,000 days period from conception. During this journey, early nutrition plays a major role in regulating developmental programming. This phase of development possibly decides the individual susceptibility to diseases like obesity, diabetes, cardiovascular diseases (CVDs), and other chronic non-communicable conditions that may occur later in life [29]. In this 
Fig. 1 Microbial dysbiosis and diseases. CKD-Chronic kidney diseases, IBD-Inflammatory bowel diseases, NAFLD-Nonalcoholic fatty liver diseases

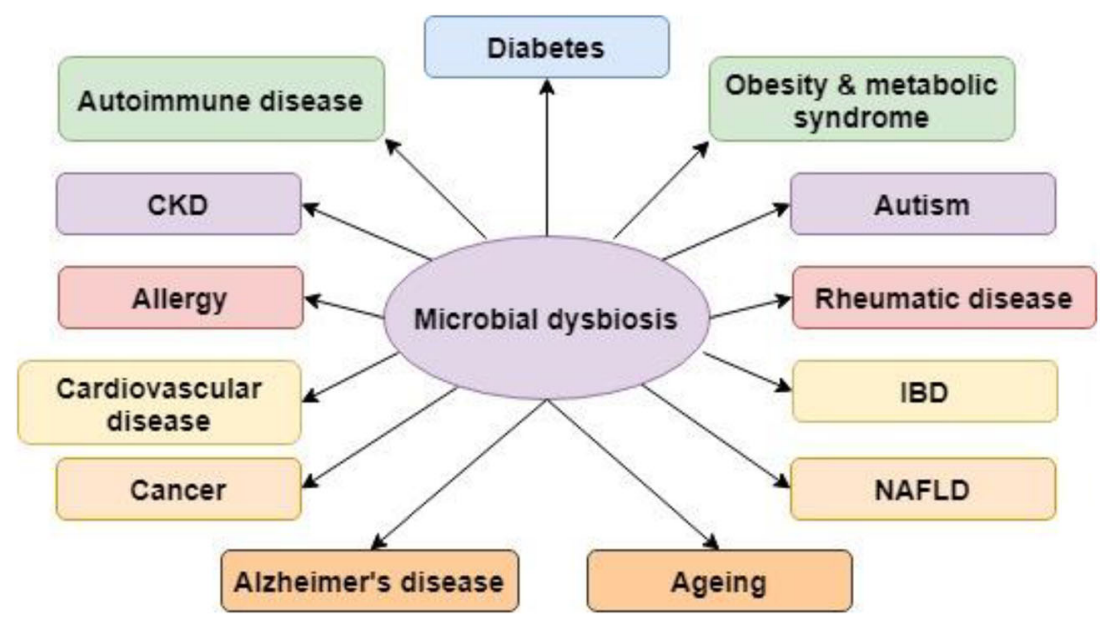

review, we describe the interactive roles of the microbiome and epigenetic regulation on human health.

\section{Gut Microbiome and Health}

Glycaemia is controlled through the gut-brain axis. Insulin resistance and hyperglycaemia are associated with disturbed communication between the enteric nervous system, and hypothalamus to the nutritional state [30]. Bacterial strains belonging to Clostridium, Bifidobacterium, Lactobacillus, Faecalibacterium, Roseburia, and Escherichia coli are involved in patients with Diabetes. Faecalibacterium and Roseburia produce short-chain fatty acids (SCFAs) such as butyrate, which play a significant role in differentiating $\mathrm{T}_{\text {reg }}$ cells and exhibit anti-inflammatory properties [30, 31]. The altered intestinal flora during Chronic kidney diseases (CKDs) exhibit an abundance of Proteobacteria, Verrucomicrobia, and Fusobacteria, leading to the elevated levels of lipopolysaccharide (LPS) and inflammation associated with the increased levels of C-reactive protein, interleukin-6, and tumor necrosis factor- $\alpha$ in serum [32]. In neurodevelopment disorders (autism which affects one out of 59 children in the USA), many functions get impaired due to microbiome dysbiosis such as maintenance of intestinal epithelial cell's tight junctions, elimination of waste and toxins, absorption of nutrition, food metabolization, production of neurotransmitters, and regulation of the immune system, and prevention of the gut colonization by pathogenic bacteria [33]. Epigenetic dysregulation is an important factor in the autism etiology, representing the effect of drugs, environment, and food on the intestinal microbiome [34]. Alzheimer's disease (neurodegenerative) is mediated by the enhanced permeability of the blood-brain barrier and the gut due to microbiota dysbiosis through signalling pathways and cytokines [35].
Epigenetic is defined as the heritable sequence-independent DNA changes and operates through histone modification (methylation, phosphorylation, and acetylation), DNA methylation, and micro-RNA-based mechanisms [36]. It plays a prominent role in the pathogenesis of CVDs, including cardiomyopathy, cardiac fibrosis, congenital heart disease, atherosclerosis, hypertension, and heart failure. The imbalance of gut microbiota leads to obesity (metabolic diseases) involving the enhanced activity of the endocannabinoid system, increased levels of LPS, and the abundance of carbohydrate fermenting bacteria [37]. Alterations in gut microbiome led to Inflammatory Bowel diseases (IBD, affects 3.5 million people) due to a reduction in SCFA producing obligate anaerobes and a rise in facultative anaerobes (E. coli) [38]. The microbiome interactions with the host play an important role in the pathogenesis of non-alcoholic fatty liver diseases (NAFLD) [39].

\section{Epigenetic Alterations and Microbiome}

DNA methylation is one of the most ubiquitous and fundamental mechanisms of epigenetics alteration that occurs on $\mathrm{CpG}$ island (5'-C-p-G-3') by DNA methyltransferases (DNMTs). DNMTs are highly susceptible to nutrient availability and are influenced by gut microbial metabolism [40]. The silencing of the estrogen receptors (ER $\alpha$, and $\operatorname{Er} \beta$ ) due to DNA methylation, results in atherosclerosis. 5-aza-2-deoxycytidine (demethylating agent) up regulated the expression of COL15A1, ER $\alpha$, and ER $\beta$.Resveratrol up regulated the expression of sirtuins (SIRT1- silent mating type information regulation type 2 homolog 1), and Acetylsalicylic acid targets ABCA1 via gene-specific DNA methylation. Through global DNA methylation, Cocoa down regulates DNMTs and methylenetetrahydrofolate reductase, and folic acid regulates multiple gene 
expression. These compounds are associated with the therapy of atherosclerosis and coronary heart disease [41].

Around 28,000 genes have been mapped for long noncoding RNAs (lncRNAs, > 200nucleotides).Like master transcription factors most of the lncRNAs show tissuespecific expression patterns, also controls diverse operations involving metabolism, nuclear organization, embryogenesis and development, differentiation, X-inactivation, proliferation/cell cycle progression, epigenetic and transcriptional regulation[42]. Out of 2000 miRNAs recognized in humans, 150-200 are associated with CVDs. The presence of miRNAs in feces of humans and mice could be considered as an indicator of intestinal malignancies. The bacterial genes usually get influenced by miRNA (an inverse correlation between the concentration of microRNA and microbial density).Microbiota imbalance-miR-181 axis has a key role in the development of insulin resistance and obesity [42].

Ageing related CVDs involve telomere attrition, dysregulated nutrient sensing, stem cell exhaustion, genomic instability, mitochondrial dysfunction, epigenetic alterations, altered intercellular communication, loss of proteostasis, and cellular senescence [43].Gut microbes metabolize choline and L-carnitine into trimethylamine- $N$ oxide (TMAO) via TMA by liver flavinmonooxygenase 3 . The evidence of links between CVD to gut microbiota via TMAO is considered as a novel opportunity for therapeutic intervention for hypertension, heart failure, stroke, CKDs, Alzheimer's, platelet aggregation, obesity, Type-1 and Type- 2 diabetes, and atherosclerosis through the uses of TMAO inhibitor [43, 44]. The sirtuins 1 and 6 (SIRT1 and 6) have a strong influence on DNA methylation and play a vital role in chromatin structure modulation through histone deacetylation. SIRT1enhances endothelium-dependent vasorelaxation through deacetylation of endothelial nitric oxide synthase and plays a beneficial role against cardiovascular ageing by promoting autophagy and by suppressing oxidative stress and inflammation [45]. Senescence-accelerated mouse prone 8 is one of the most suitable models to study non-chronological and natural vascular ageing. Ceramides get accumulated/enhanced during ageing and age-related stress conditions, thus involved in cell cycle arrest/apoptotic signalling. During ageing, chronic cellular damage results in over cargo accumulation or due to diminished autophagic flux making autophagy insufficient causing the interacting signals between gut microbiota, ageing, and sphingolipids [46].

\section{Microbiota Based Health Interventions}

Protein sources (casein, soy, cod, beef, chicken, and pork) modulate energy efficiency, and obesity progression by affecting gut microbiota. The terrestrial animal-based proteins have been found to be more obesogenic than those of seafood or vegetables [26, 47]. Human and animal-based studies have suggested that the level of Lactobacilli and Bifidobacterium can be increased after consuming soy foods and is beneficial for reducing the risk of diseases. Metabolites (betaine, folate, choline, and vitamin $\mathrm{B}_{12}$ ) are potentially implicated in the synthesis of 6-methyltetrahydrofolate (methyl donor) to generate S-adenosylmethionine, which participates in DNA methylation [47].The gut metabolites like folate, acetate, and butyrate are responsible for epigenetic modifications through the regulation of various enzyme activities. The SCFA, butyrate is known to be a potent histone deacetylase (HDAC) inhibitor which promotes histone hyper acetylation and enhances the accessibility for gene transcription. Butyrate-based HDAC inhibition triggered its anti-inflammatory property by repressing nuclear factor $\kappa \mathrm{B}$ and interferon $\gamma$ production, and by enhancing peroxisome proliferator-activated receptor $\gamma$ (PPAR $\gamma$ ) expression in colon cancer. PPAR $\gamma$ is protective against cardiac diseases, therefore butyrate and its sub-products like sodium butyrate acts as an anti-inflammatory agent against CVDs. Butyrate has another protective potential which operates by histone $\mathrm{H}_{3}$ modification which alters G1-specific cell cycle proteins leading to the arrest of the proliferation of smooth muscle cells and induces the production of regulatory $\mathrm{T}$ cells in the colon to boost the immune system [47].

Probiotics (living microorganisms), which when given out in sufficient amounts confer health benefits on the host. Lactobacilli containing fermented milk products have been reported for the longevity of Bulgarians. The mechanism of probiosis comprises stimulation of epithelial cell proliferation, immunomodulation, manipulation of intestinal microbial communities, differentiation, and fortification of the intestinal barrier, and the suppression of pathogens [48]. Faecal microbiota transplantation carries therapeutic potential against functional gastrointestinal disorders, obesity, metabolic syndromes, and inflammatory bowel disease through the administration of faecal microbiota into the recipient's intestinal tract from a healthy donor. This transplantation changes the microbial composition of the gut of the recipient and makes them healthier [49]. Many drugs are playing the bidirectional communication with the microbiome. Known drugs in case of diabetes that interact with the microbiome include anti-diabetic drugs like metformin, a thiazolidinedione, inulin-type fructans, dipeptidyl peptidase-4 inhibitors, glucagon-like peptide-1 receptor, 
andalpha-glucosidase inhibitor. Gut microbiota composition studies suggested that increased frequency of moderate exercise from never to daily enhances diversity among the phylum firmicutes (including Faecalibacteriumprausnitzii, and species from the genus Coprococcus, Lachnospira, and Oscillospira) that promotes a healthier environment in the gut. Regular physical exercise associates strongly with cardiovascular health benefits during ageing through the mechanism of epigenetic modifications [50].

\section{Conclusion}

Gut microbiome and host bidirectional communications play an important role in the prevention and treatment of many diseases like diabetes, autism, Alzheimer's, CVDs, CKD, IBD, obesity, fatty liver, and lung diseases. The detailed works on microbes and their metabolic products are expected to modulate the associations of microbes.

Acknowledgement The authors received no funding for this work.

\section{Complaince with Ethical Standards}

Conflict of interest The authors declare no conflict of interest.

\section{References}

1. Qin Y, Wade PA (2018) Crosstalk between the microbiome and epigenome: messages from bugs. J Biochem 163:105-112. https://doi.org/10.1093/jb/mvx080

2. Kumar R, Sood U, Gupta V, Singh M, Scaria J, Lal R (2020) Recent advancements in the development of modern probiotics for restoring human gut microbiome dysbiosis. Indian J Microbiol 60:12-25. https://doi.org/10.1007/s12088-019-00808-y

3. Kumar A, Jain SR, Sharma CB, Joshi AP, Kalia VC (1995) Increased $\mathrm{H}_{2}$ production by immobilized microorganisms. World J Microbiol Biotechnol 11:156-159. https://doi.org/10.1007/ BF00704638

4. Kumar P, Patel SKS, Lee J-K, Kalia VC (2013) Extending the limits of Bacillus for novel biotechnological applications. Biotechnol Adv 31:1543-1561. https://doi.org/10.1016/j.bio techadv.2013.08.007

5. Lee J-K, Patel SKS, Sung BH, Kalia VC (2020) Biomolecules from municipal and food industry wastes: an overview. Bioresour Technol 298:122346. https://doi.org/10.1016/j.biortech.2019. 122346

6. Patel SKS, Gupta RK, Das D, Lee J-K, Kalia VC (2020) Continuous biohydrogen production from poplar biomass hydrolysate by a defined bacterial mixture immobilized on lignocellulosic materials under non-sterile conditions. J Clean Prod. https://doi. org/10.1016/j.jclepro.2020.125037

7. Patel SKS, Lee J-K, Kalia VC (2018) Nanoparticles in biological hydrogen production: An overview. Indian J Microbiol 58:8-18. https://doi.org/10.1007/s12088-017-0678-9

8. Patel SKS, Ray S, Prakash J, Wee JH, Kim S-Y, Lee J-K, Kalia VC (2019) Co-digestion of biowastes to enhance biological hydrogen process by defined mixed bacterial cultures. Indian $\mathrm{J}$
Microbiol 59:154-160. https://doi.org/10.1007/s12088-01800777-8

9. Kondaveeti S, Patel SKS, Pagolu R, Li J, Kalia VC, Choi M-S, Lee J-K (2019) Conversion of simulated biogas to electricity: Sequential operation of methanotrophic reactor effluents in microbial fuel cell. Energy 189:116309. https://doi.org/10.1016/j. energy.2019.116309

10. Patel SKS, Gupta RK, Kumar V, Kondaveeti S, Kumar A, Das D, Kalia VC, Lee J-K (2020) Biomethanol production from methane by immobilized cocultures of methanotrophs. Indian J Microbiol 60:318-324. https://doi.org/10.1007/s12088-020-00883-6

11. Otari SV, Patel SKS, Kalia VC, Kim I-W, Lee J-K (2019) Antimicrobial activity of biosynthesized silver nanoparticles decorated silica nanoparticles. Indian J Microbiol 59:379-382. https://doi.org/10.1007/s12088-019-00812-2

12. Patel SKS, Kim J-H, Kalia VC, Lee J-K (2019) Antimicrobial activity of amino-derivatized cationic polysaccharides. Indian $\mathrm{J}$ Microbiol 59:96-99. https://doi.org/10.1007/s12088-018-00764-7

13. Sajid A, Arora G, Singhal A, Kalia VC, Singh Y (2015) Protein phosphatases of pathogenic bacteria: role in physiology and virulence. Annu Rev Microbiol 69:527-547. https://doi.org/10.1146/ annurev-micro-020415-111342

14. Kalia VC, Patel SKS, Kang YC, Lee J-K (2019) Quorum sensing inhibitors as antipathogens: biotechnological applications. Biotechnol Adv 37:68-90. https://doi.org/10.1016/j.biotechadv. 2018,11(006),pp.67

15. Meena H, Mishra R, Ranganathan S, Sarma VV, Ampasala DR, Kalia VC, Lee J-K, Siddhardha S (2020) Phomopsis tersa as inhibitor of quorum sensing systems and biofilm forming ability of Pseudomonas aeruginosa. Indian J Microbiol 60:70-77. https://doi.org/10.1007/s12088-019-00840-y

16. Parasuraman P, Devadatha B, Sarma VV, Ranganathan S, Ampasala DR, Reddy D, Kumavath R, Kim I-W, Patel SKS, Kalia VC, Lee J-K, Siddhardha B (2020) Inhibition of microbial quorum sensing mediated virulence factors by Pestalotiopsis sydowiana. J Microbiol Biotechnol 30:571-582. https://doi.org/ 10.4014/jmb.1907.07030

17. Ray S, Patel SKSP, Singh M, Singh GP, Kalia VC (2019) Exploiting polyhydroxyalkanoates for tissue engineering. In: Kalia VC (ed) Biotechnological applications of polyhydroxyalkanoates. Springer, Singapore, pp 271-282

18. Kalia VC, Ray S, Patel SKSP, Singh M, Singh GP (2019) Applications of polyhydroxyalkanoates and their metabolites as drug carriers. In: Kalia VC (ed) Biotechnological applications of polyhydroxyalkanoates. Springer, Singapore, pp 35-48

19. Bajaj A, Purohit HJ (2020) Understanding SARS-CoV-2: genetic diversity, transmission and cure in human. Indian $\mathrm{J}$ Microbiol 60:398-401. https://doi.org/10.1007/s12088-020-00869-4

20. Ha K-M (2020) A lesson learned from the outbreak of COVID-19 in Korea. Indian J Microbiol 60:396-397. https://doi.org/10.1007/ s12088-020-00882-7

21. Patel SKS, Lee JK, Kalia VC (2020) Deploying biomolecules as anti-COVID-19 agents. Indian J Microbiol 60(3):263-268. https://doi.org/10.1007/s12088-020-00893-4

22. Rishi P, Thakur K, Vij S, Rishi L, Singh A, Kaur IP, Patel SKS, Lee J-K, Kalia VC (2020) Diet, Gut Microbiota and COVID-19. Indian J Microbiol 60:420-429. https://doi.org/10.1007/s12088020-00908-0

23. Nile SH, Kai G (2021) Recent clinical trials on natural products and traditional Chinese medicine combating the COVID- 19 . Indian J Microbiol 61:19-25. https://doi.org/10.1007/s12088020-00919-X

24. Yu L (2021) Restoring good health in elderly with diverse gut microbiome and food intake restriction to combat COVID-19. Indian J Microbiol 61:114-117. https://doi.org/10.1007/s1208801920-00913-3 
25. Kalia VC (2014) Microbes, antimicrobials and resistance: the battle goes on. Indian J Microbiol 54:1-2. https://doi.org/10. 1007/s12088-013-0443-7

26. Sood U, Gupta V, Kumar R, Lal S, Fawcett D, Rattan S, Poinern GEJ, Lal R (2020) Chicken gut microbiome and human health: past scenarios, current perspectives, and futuristic applications. Indian J Microbiol 60:2-11. https://doi.org/10.1007/s12088-01900785-2

27. Whayne TF (2015) Epigenetics in the development, modification, and prevention of cardiovascular disease. Mol Biol Rep 42:765-776. https://doi.org/10.1007/s11033-014-3727-z

28. Sender R, Fuchs S, Milo R (2016) Are we really vastly outnumbered? revisiting the ratio of bacterial to host cells in humans. Cell 164:337-340. https://doi.org/10.1016/j.cell.2016. 01.013

29. Indrio F, Martini S, Francavilla R, Corvaglia L, Cristofori F, Mastrolia SA, Neu J, Rautava S, Spena GR, Raimondi F, Loverro G (2017) Epigenetic matters: The link between early nutrition, microbiome, and long-term health development. Front Pediatr 5:178. https://doi.org/10.3389/fped.2017.00178

30. Bessac A, Cani PD, Meunier E, Dietrich G, Knauf C (2018) Inflammation and gut-brain axis during type 2 diabetes: Focus on the crosstalk between intestinal immune cells and enteric nervous system. Front Neurosci 12:725. https://doi.org/10.3389/fnins. 2018.00725

31. Sharma M, Li Y, Stoll ML, Tollefsbol TO (2020) The epigenetic connection between the gut microbiome in obesity and diabetes. Front Genet 10:1329. https://doi.org/10.3389/fgene.2019.01329

32. Salguero M, Al-Obaide M, Singh R, Siepmann T, Vasylyeva TL (2019) Dysbiosis of Gram-negative gut microbiota and the associated serum lipopolysaccharide exacerbates inflammation in type 2 diabetic patients with chronic kidney disease. Exp Ther Med 18:3461-3469. https://doi.org/10.3892/etm.2019.7943

33. Tonacci A, Bagnato G, Pandolfo G, Billeci L, Sansone F, Conte R, Gangemi S (2019) MicroRNA cross-involvement in autism spectrum disorders and atopic dermatitis: a literature review. J Clin Med 8:88. https://doi.org/10.3390/jcm8010088

34. Eshraghi RS, Deth RC, Mittal R, Aranke M, Kay SS, Moshiree B, Eshraghi AA (2018) Early disruption of the microbiome leading to decreased antioxidant capacity and epigenetic changes: Implications for the rise in autism. Front Cell Neurosci 12:256. https://doi.org/10.3389/fncel.2018.00256

35. Jiang C, Li G, Huang P et al (2017) The gut microbiota and Alzheimer's disease. J Alzheimer's Dis 58:1-15. https://doi.org/ 10.3233/JAD-161141

36. Metzinger L, De Franciscis S, Serra R (2017) The management of cardiovascular risk through epigenetic biomarkers. Biomed Res Int 2017:9158572. https://doi.org/10.1155/2017/9158572

37. Muscogiuri G, Cantone E, Cassarano S, Tuccinardi D, Barrea L, Savastano S, Colao A (2019) Gut microbiota: a new path to treat obesity. Int J Obes Suppl 9:10-19. https://doi.org/10.1038/ s41367-019-0011-7
38. Verma H, Phian S, Lakra P, Kaur J, Subudhi S, Lal R, Rawat CD (2020) Human gut microbiota and mental health: advancements and challenges in microbe-based therapeutic interventions. Indian J Microbiol 60:405-419. https://doi.org/10.1007/s12088-02000898-z

39. Bashiardes S, Shapiro H, Rozin S, Shibolet O, Elinav E (2016) Non-alcoholic fatty liver and the gut microbiota. Mol Metab 5:782-794. https://doi.org/10.1016/j.molmet.2016.06.003

40. Prasher D, Greenway SC, Singh RB (2019) The impact of epigenetics on cardiovascular disease. Biochem Cell Biol 98:12-22. https://doi.org/10.1139/bcb-2019-0045

41. Zarzour A, Kim HW, Weintraub NL (2019) Epigenetic regulation of vascular diseases. Arterioscler Thromb Vasc Biol 39:984-990. https://doi.org/10.1161/atvbaha.119.312193

42. Kura B, Parikh M, Slezak J, Pierce GN (2019) The influence of diet on microRNAs that impact cardiovascular disease. Molecules 24:1509. https://doi.org/10.3390/molecules24081509

43. Canyelles M, Tondo M, Cedó L, Farràs M, Escolà-Gil JC, Blanco-Vaca F (2018) Trimethylamine N-oxide: A link among diet, gut microbiota, gene regulation of liver and intestine cholesterol homeostasis and HDL function. Int J Mol Sci 19:3228. https://doi.org/10.3390/ijms19103228

44. Ke Y, Li D, Zhao M et al (2018) Gut flora-dependent metabolite Trimethylamine-N-oxide accelerates endothelial cell senescence and vascular aging through oxidative stress. Free Radic Biol Med 116:88-100. https://doi.org/10.1016/j.freeradbiomed.2018.01.007

45. Vikram A, Kim YR, Kumar S, Li Q, Kassan M, Jacobs JS, Irani K (2016) Vascular microRNA-204 is remotely governed by the microbiome and impairs endothelium-dependent vasorelaxation by downregulating Sirtuin1. Nat Commun 7:12565. https://doi. org/10.1038/ncomms 12565

46. Arboleda G, Morales LC, Benítez B, Arboleda H (2009) Regulation of ceramide-induced neuronal death: Cell metabolism meets neurodegeneration. Brain Res Rev 59:333-346. https://doi. org/10.1016/j.brainresrev.2008.10.001

47. Madsen L, Myrmel LS, Fjære E, Liaset B, Kristiansen K (2017) Links between dietary protein sources, the gut microbiota, and obesity. Front Physiol 8:1047. https://doi.org/10.3389/fphys. 2017.01047

48. Hemarajata P, Versalovic J (2013) Effects of probiotics on gut microbiota: Mechanisms of intestinal immunomodulation and neuromodulation. Therap Adv Gastroenterol 6:39-51. https://doi. org/10.1177/1756283X12459294

49. Gupta S, Allen-Vercoe E, Petrof EO (2016) Fecal microbiota transplantation: In perspective. Therap Adv Gastroenterol 9:229-239. https://doi.org/10.1177/1756283X15607414

50. Mach N, Fuster-Botella D (2017) Endurance exercise and gut microbiota: A review. J Sport Health Sci 6:179-197. https://doi. org/10.1016/j.jshs.2016.05.001

Publisher's Note Springer Nature remains neutral with regard to jurisdictional claims in published maps and institutional affiliations. 